\title{
Simulations of Noise in Disordered Systems
}

\author{
C. J. Olson Reichhardt ${ }^{a}$ and C. Reichhardt ${ }^{b}$ \\ ${ }^{a} \mathrm{~T}-12$, Los Alamos National Laboratory, Los Alamos, NM, USA \\ ${ }^{b}$ T-CNLS, Los Alamos National Laboratory, Los Alamos, NM, USA
}

\begin{abstract}
We use particle dynamics simulations to probe the correlations between noise and dynamics in a variety of disordered systems, including superconducting vortices, 2D electron liquid crystals, colloids, domain walls, and granular media. The noise measurements offer an experimentally accessible link to the microscopic dynamics, such as plastic versus elastic flow during transport, and can provide a signature of dynamical reordering transitions in the system. We consider broad and narrow band noise in transport systems, as well as the fluctuations of dislocation density in a system near the melting transition.
\end{abstract}

Keywords: superconducting vortices, colloids, voltage noise, melting

\section{INTRODUCTION}

Noise measurements have found fruitful applications as a tool to probe both equilibrium and nonequilibrium transitions. An open question is whether phase transitions of the type observed in equilibrium systems can also occur in nonequilibrium systems, or whether it is even meaningful to refer to "phases" in the latter case. A particularly interesting issue is whether there are similarities between the melting transition of a thermal system and the disordered or plastic flow that occurs in a system driven out of equilibrium over quenched disorder. In this paper, we explore some suggestive similarities in the noise properties of equilibrium and nonequilibrium systems, based on our simulation studies ${ }^{1-6}$ of a wide range of physical systems. We are particularly interested in possible connections between the dynamical heterogeneities observed above the melting transition in a thermal system, and those found in the plastic or disordered flow state of a driven system.

We begin by considering the noise produced during the melting of a vortex system confined to one dimension. We compare this to the case of a colloid system melting in two dimensions, and find that similar features appear in the noise. Finally, we consider a driven system in the presence of quenched disorder, and show that as the driving force is decreased, similar noise signatures appear as when the temperature is increased in the thermal systems. We identify the heterogeneities responsible for the noise in each case, and suggest how noise signatures can be used to further probe these systems and increase our understanding of both melting and plasticity.

Since this paper does not focus on a single system, but refers to work performed on several different physical systems, we offer some very brief background information here. Ideal superconductors carry current without resistance and perfectly expel externally applied magnetic fields. The superconductivity is destroyed when too great a magnetic field is applied, and the material becomes a normal resistive conductor. Type-II materials remain superconducting in high magnetic fields by allowing the magnetic flux to penetrate the material in the form of discrete quantized vortices which repel each other and interact with defects in the superconducting material. The superconducting material returns to its normal resistive state only at the center of these vortices; the remainder of the material still carries a supercurrent. The vortices experience a Lorentz force from the flowing current and move through the superconductor until they are trapped, or pinned, at defect sites. Another system of repulsive, overdamped particles which shows many of the same dynamical features as the vortex system is colloids, which are micron-sized particles suspended in a liquid solution. Each colloidal particle carries an electrostatic charge, leading to a repulsive interaction between the colloids with a range that can be adjusted by varying the salt concentration of the solution. The colloids can be confined to two dimensions by means of laser trapping, and can interact with quenched disorder which may take the form of optical traps or a structured surface.

Further author information:

C.J.O.R.: E-mail: cjrx@lanl.gov, Telephone: 15056651134

C.R.: E-mail: charlesr@cnls.lanl.gov, Telephone: 15056650059 


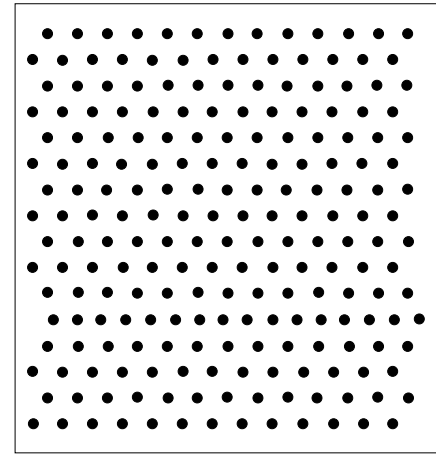

(a)

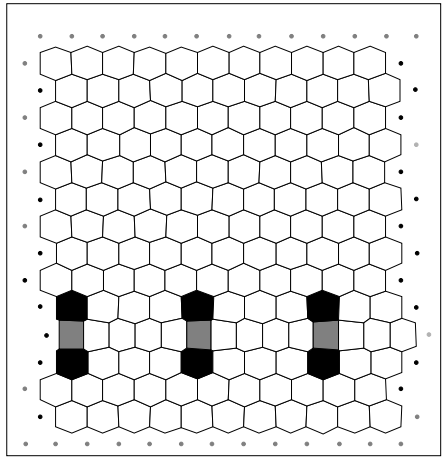

(b)

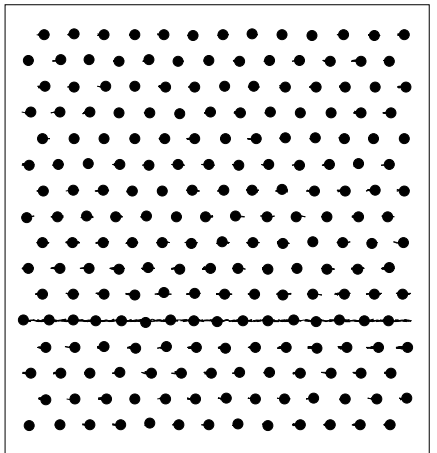

(c)

Figure 1. (a) Black dots: Vortices in the vortex chain sample. The 1D channel of increased vortex density is in the lower third of the panel. (b) Voronoi construction indicating sixfold (white), sevenfold (black), and fourfold (gray) coordinated vortices. The three extra vortices inside the channel each form a defect. (c) Lines indicate vortex motion in the system for a temperature $T / T_{m}^{B}=0.25$ above the transition to motion along the chain.

\section{NOISE OF ONE-DIMENSIONAL MELTED SYSTEMS}

To explore noise signatures near a melting transition, we begin with the simplest case of a system confined to a one-dimensional (1D) channel. Such a system can be created artificially by, for example, using an optical trap to confine colloids to a $1 \mathrm{D}$ line, ${ }^{7}$ or by nanofabricating channels in a superconducting material, which then serve to confine superconducting vortices. ${ }^{8-12}$ Quasi-1D confinement of vortices can also arise naturally due to trapping by extended defects such as grain ${ }^{13,14}$ and twin boundaries. ${ }^{15}$ Additionally, when a tilted field is applied to layered superconductors, vortex chains appear in which a portion of the vortices align in a string with a smaller lattice spacing than that of the bulk vortices. ${ }^{16}$ We choose to model the vortex chain system as an example of $1 \mathrm{D}$ confined melting.

For all of the systems described in this paper, we perform numerical simulations of overdamped particles in a two-dimensional (2D) sample with periodic boundary conditions in the $x$ and $y$ directions. For the vortex chain state, we model the vortices as $N_{v}$ repulsive particles which interact with weak random quenched disorder and thermal noise. We confine a portion of the vortices to move along a quasi-1D channel. The equation of motion for a vortex $i$ is

$$
\eta \mathbf{v}=\mathbf{f}_{i}=-\sum_{j \neq i}^{N_{v}} \nabla_{i} U\left(r_{i j}\right)+\mathbf{f}_{i}^{p}+\mathbf{f}_{i}^{T} .
$$

Here $\eta=1$ is the damping constant and $U_{v}=-\ln (r)$ is the vortex interaction potential, treated as in Ref. ${ }^{17}$ The pinning potential arises from $N_{p}$ randomly spaced attractive parabolic traps of range $r_{p}$ and strength $f_{p}=1.0$. The thermal noise $\mathbf{f}_{i}^{T}$ arises from random Langevin kicks with $<f^{T}(t)>=0$ and $<f_{i}^{T}(t) f_{j}^{T}\left(t^{\prime}\right)>=$ $2 \eta k_{B} T \delta_{i j} \delta\left(t-t^{\prime}\right)$ We measure temperature in units of the temperature at which the bulk vortices melt, $T_{m}$. Further details of the simulation can be found in Ref. ${ }^{4}$

To prepare the system, we place a triangular array of vortices with lattice constant $a$ in the sample, and then put additional vortices into the 1D confining channel, so that the lattice constant inside the channel $a^{\prime}<a$, as illustrated in Fig. 1(a). In order to accommodate the incommensuration between the vortices in the channel and the surrounding vortex lattice, dislocations form along the channel. These can be imaged by performing a Voronoi tessellation of the sample, as shown in Fig. 1(b). At low temperatures, the incommensuration in the chain is stationary or pinned in a well defined location. As the temperature increases, the vortices inside the chain become mobile at temperatures well below the bulk melting transition $T_{m}^{B}$ of the triangular lattice, and begin to move back and forth along the channel, as indicated in Fig. 1(c). The transition from localized to mobile vortices inside the channel can be regarded as a $1 \mathrm{D}$ melting transition. 


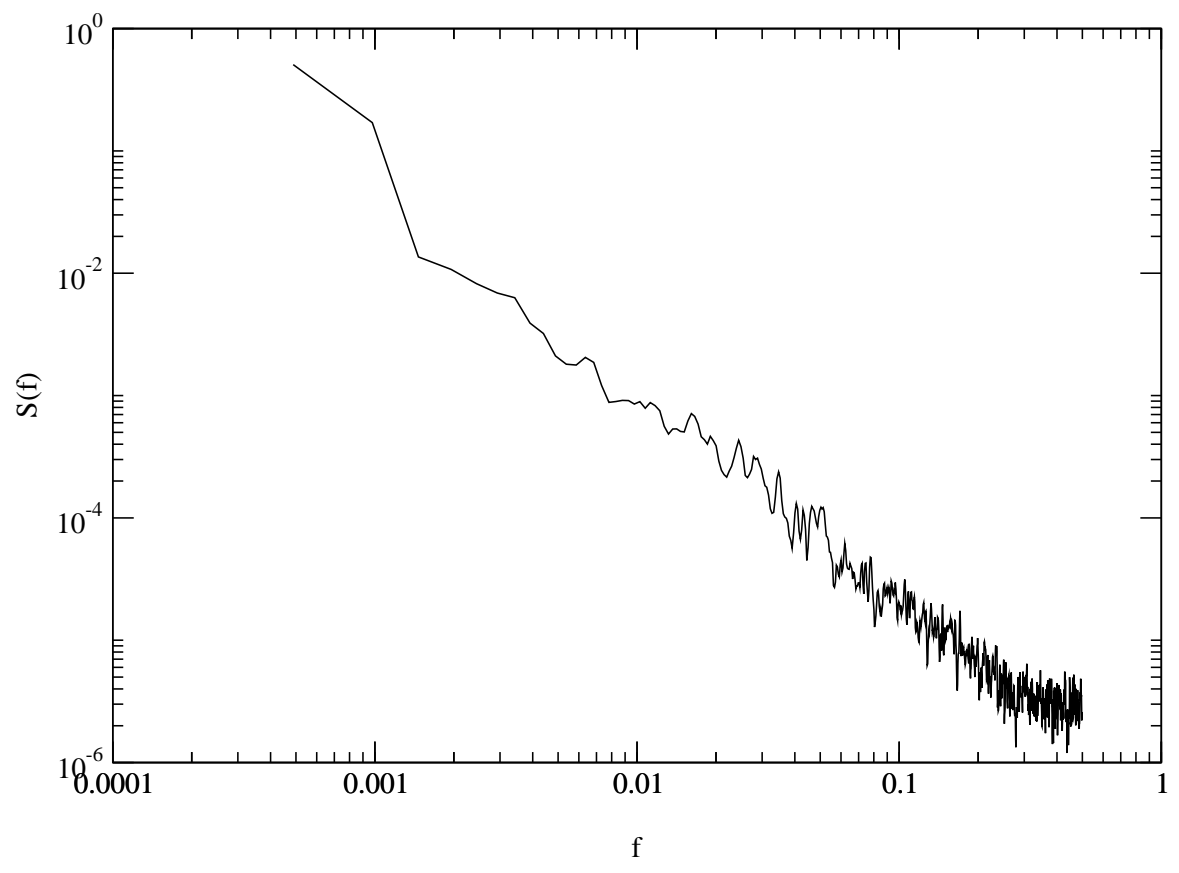

Figure 2. The power spectra $S(f)$ for the displacement of the vortex chain $X$ at a temperature just above melting, $T / T_{m}^{B}=0.25$. The spectra has a $1 / f^{\alpha}$ form, with $\alpha \sim 1.6$.

As a measure of the vortex motion along the chain at a given temperature, we consider the net displacement $X$ of a vortex in the chain from its starting position. For temperatures just above where the vortex chain becomes mobile, individual vortices along the chain do not move continuously, but instead move in discrete jumps of nearly a lattice constant in magnitude. These jumps run through the chain as a soliton like pulse with the vortices jumping sequentially. The size of the jumps is determined by the periodic potential created by the stationary ordered bulk vortices. The power spectra $S(f)=\left|\int X(t) e^{-2 \pi i f t} d t\right|^{2}$ of the single vortex motion just above melting has a $1 / f^{\alpha}$ signature, with $\alpha=1.6$, as shown in Fig. 2. This results from the discrete nature of the jumps in $X$. As the temperature further increases, the motion along the chain becomes more continuous, and the magnitude of low frequency power in $S(f)$ drops until, at high temperatures, the noise spectra becomes white. This indicates that a noise signature can be used to probe how correlated the fluctuations in the system are at temperatures close to and well above melting.

Note that the soliton-like pulse, as it moves through the sample, causes an individual vortex to abruptly shift its position by approximately a lattice constant. Thus, in monitoring the position of one vortex in the chain, we observe relatively long periods when the vortex is essentially stationary, separated by very rapid motion to a new position as the soliton sweeps past. It may be more useful to think of the soliton itself as the "particle" moving through the system, since the soliton is diffusing continuously through the chain. The solitons can be directly identified by the 7-4-7 trios of dislocated vortices seen in Fig. 1(b). Note also that the solitons or dislocations, which are not constrained to remain fixed to a given vortex, can move through the system much more rapidly than individual vortices can.

\section{NOISE OF TWO-DIMENSIONAL MELTED SYSTEMS}

We next turn to melting in a $2 \mathrm{D}$ system. There is much previous work in this area, and a considerable number of studies have focused on dynamical heterogeneities which occur at temperatures above melting when certain regions of the sample have a higher mobility than the rest of the sample. ${ }^{18-24}$ These heterogeneities are both 


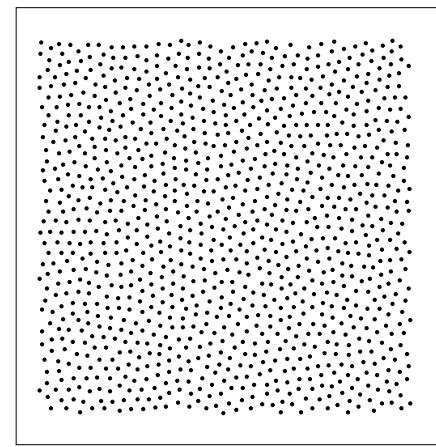

(a)

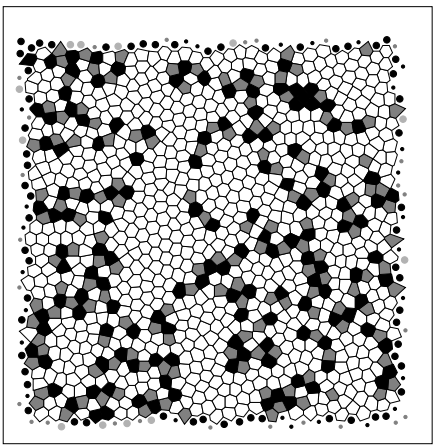

(b)

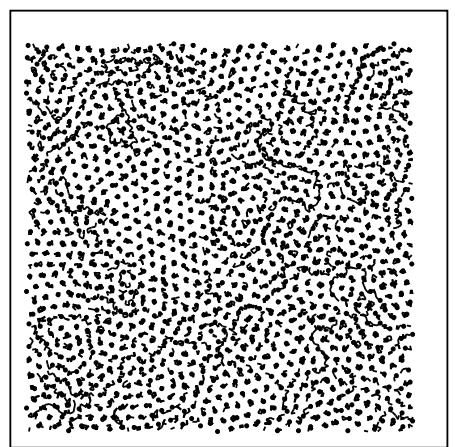

(c)

Figure 3. (a) Black dots: Colloids in the two-dimensional melting sample at $T / T_{m}=1.04$, just above the melting transition. (b) Voronoi construction indicating sixfold (white), sevenfold (black), and fivefold (dark gray) coordinated particles. (c) Lines indicate colloid motion over a fixed time interval.

temporal, such as when a group of particles moves along a stringlike structure, ${ }^{25}$ and spatial, due to the fact that the 5-7 dislocation pairs in the lattice form highly inhomogeneous distributions. ${ }^{24,26-31}$

Since we have observed interesting noise signatures related to motion along a 1D chain in the previous section, it is interesting to ask whether similar noise signatures appear for stringlike motion that is not confined to 1D. To investigate this question, we have performed simulations of colloidal particles as a specific realization of overdamped repulsive particles that form a triangular lattice in the absence of temperature. In this case, the equation of motion for the colloids is again given by Eqn. (1), but without quenched disorder, $\mathbf{f}_{i}^{p}=0$, and with a screened Coulomb interaction,

$$
U\left(r_{i j}\right)=\frac{Q^{2}}{r} \exp (-\kappa r)
$$

Here $Q$ is the charge on the colloid and $1 / \kappa$ is the screening length, which can be adjusted experimentally by varying the salt concentration of the solution. We take $\kappa=2 / a$, where $a$ is the lattice constant. In each simulation, we fix the temperature to a value above the melting transition, wait for $10^{6}$ time steps for any transients to disappear, and then begin to take data. Further details of the simulation appear in Ref. ${ }^{5}$

We illustrate the behavior of the system at two temperatures above melting in Figs. 3 and 4. Just above melting, shown in Fig. 3, about 30\% of the system is filled with defects, but these defects are not evenly spread throughout the sample. Instead, the dislocations cluster into strings, which form grain boundaries around sixfoldcoordinated regions. Particle motion is concentrated along the defect strings, as illustrated in Fig. 3(c). This motion can be regarded as arising not so much from the movement of the particles themselves, but rather as being caused by the motion of the grain boundaries or defect strings. In order for a defect string to move over by one lattice constant, the particles currently inside the defect string must shift position in order to join the ordered grain that is growing by one lattice constant as a result of the motion of the boundary. Thus, if we monitor the movement of a single particle, we observe that the particle remains stationary for a period of time before suddenly shifting position by a sizable fraction of a lattice constant. If we were, however, to instead monitor the motion of a dislocation, we would find a more continuous motion without long stationary pauses as the dislocation diffuses thermally. It may in some cases be more useful to think of the dislocations themselves as the primary "particles" whose dynamics are controlling the behavior of the system, rather than the actual colloidal particles which we are directly simulating. The dislocations are more complicated entities than the particles, however, both because they can be thermally created and destroyed, unlike the colloids, and also because they do not remain fixed to a single colloid but can move through the lattice much more rapidly than an individual particle can. There are clear analogies to the 1D case of diffusing dislocation discussed in Section 2, although switching to two dimensions has introduced considerable complexity since the lines along which the dislocations can move vary spatially. 


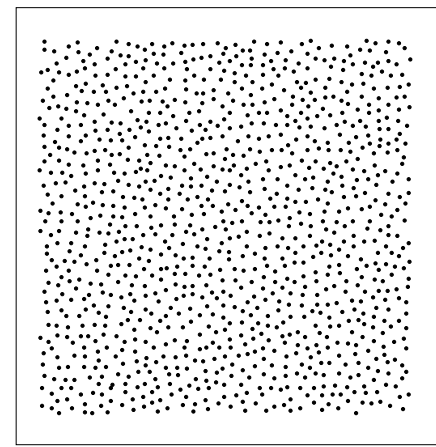

(a)

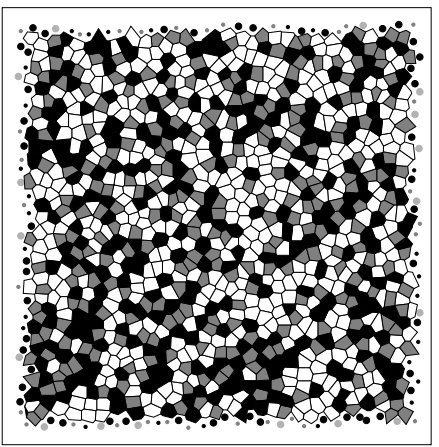

(b)

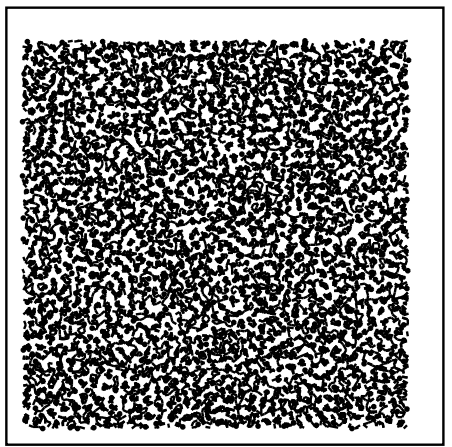

(c)

Figure 4. (a) Black dots: Colloids in the two-dimensional melting sample at $T / T_{m}=7.0$, well above the melting transition. (b) Voronoi construction indicating sixfold (white), sevenfold (black), and fivefold (dark gray) coordinated particles. (c) Lines indicate colloid motion over a fixed time interval.
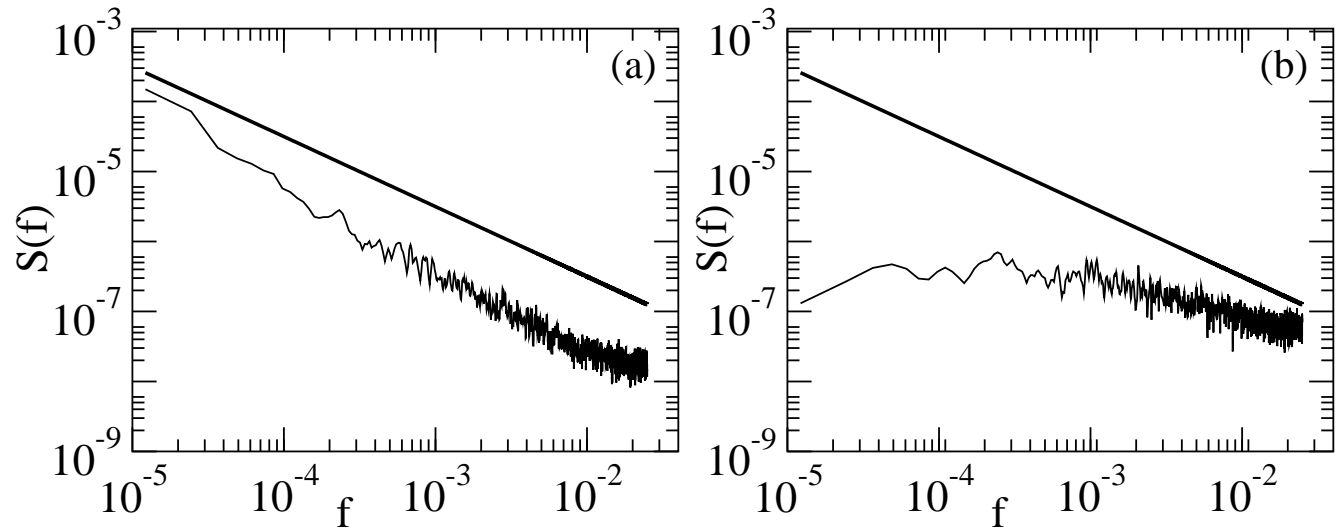

Figure 5. (a) The power spectrum for the time series of the density of dislocations in the colloid system just above melting at $T / T_{m}=1.04$. The solid line indicates a slope of $1 / f$. (b) The power spectrum for the time series at $T / T_{m}=7.0$ along with a $1 / f$ line.

When the temperature is increased to a value well above the melting transition, the number of dislocations increases to fill nearly half of the sample. As illustrated in Fig. 4, the motion of the colloids over time is now essentially homogeneous, and the system is in a liquid-like state. In this case, dislocations are being created and destroyed so rapidly and easily that their motions cannot be distinguished from those of the particles, which are now moving freely through the sample.

Since the motions of the dislocations play such a strong role in the particle displacements just above melting, we use the dislocations themselves as a means of characterizing the behaviors of the system at the different temperatures. We compute the defect configuration and density every 20 time steps for 20000 frames and obtain a time series of the defect density for several temperatures. Just above melting we observe long time fluctuations of the dislocation density, corresponding to the persistence of the chain-like structures shown in Fig. 3. In contrast, at higher temperatures well above melting, the fluctuations are very rapid. In Fig. 5(a) we plot the power spectrum $S(f)$ of $P_{6}$ for $T / T_{m}=1.04$, which fits well to a $1 / f^{\alpha}$ scaling over more than three decades with the best fit $\alpha=1.04$, close to $1 / f$ noise. As the temperature increases, the spectrum changes from $1 / f$ to white noise $(\alpha=0)$ for low frequencies, as shown in Fig. $5(\mathrm{~b})$ for $T / T_{m}=7.0$.

It is clear from Fig. 5 that the amount of power at low frequencies drops dramatically at the higher tempera- 


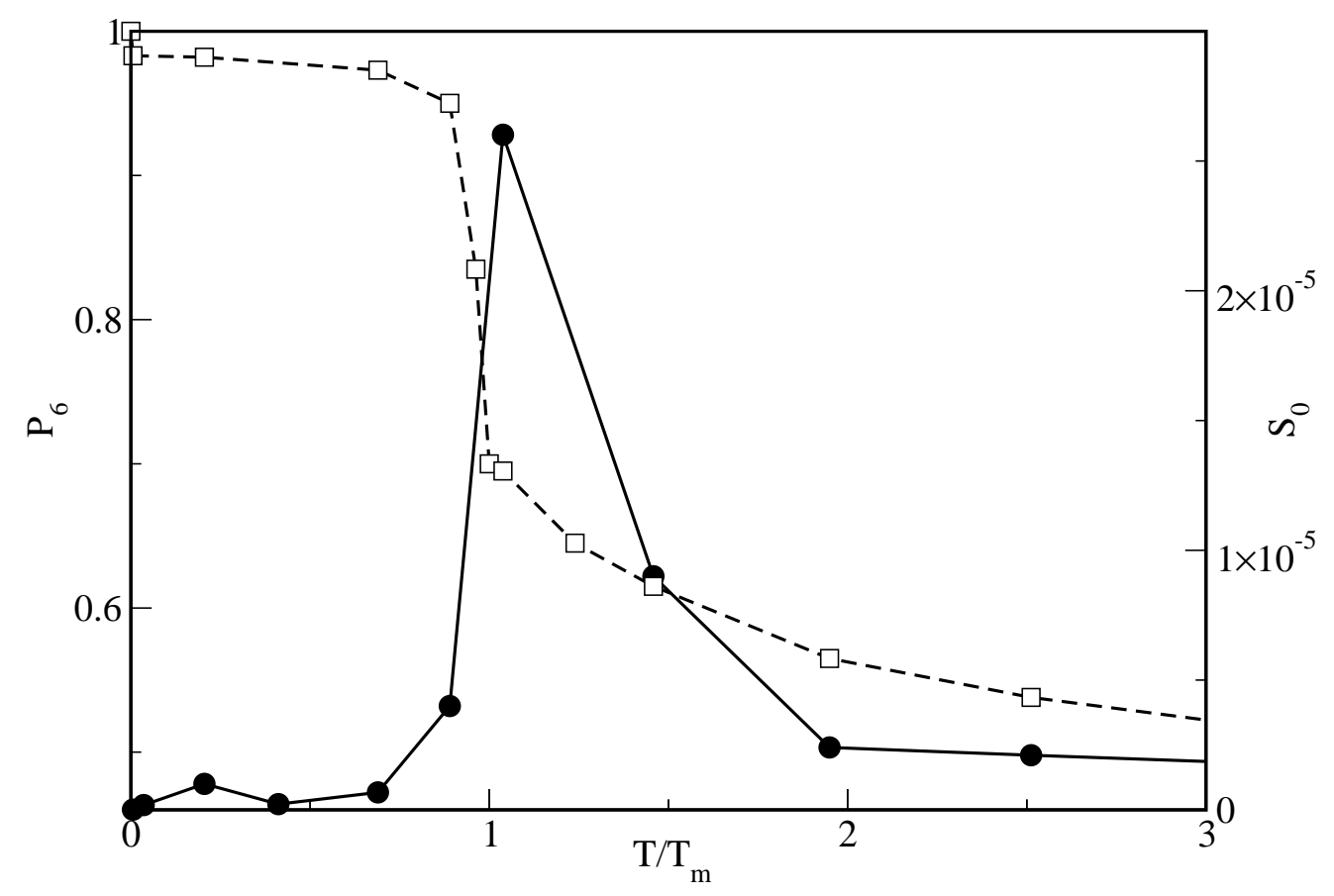

Figure 6. Squares (dotted line): The fraction of sixfold coordinated particles $P_{6}$ versus temperature, with melting occurring at $T / T_{m}=1$. Circles (solid line): The integrated noise power $S_{0}$, obtained from $S(f)$, shows a peak just above the melting transition.

ture, when long-time fluctuations in the defect density are no longer present. This suggests that the noise power could provide a useful means of characterizing the system above melting. We integrate $S(f)$ over the first octave of frequencies to obtain the noise power $S_{0},{ }^{32,33}$ which we plot in Fig. 6 as a function of temperature along with the defect density. There is a prominent peak in $S_{0}$ which coincides with the onset of the defect proliferation above melting. For increasing $T$, the noise power falls and saturates when the spectrum becomes white. Thus, just above melting, when the system is highly heterogeneous, the noise power is maximized. It is especially interesting that $S_{0}$ appears to be diverging near $T / T_{m}=1$, suggesting that $S_{0}$ may be a useful indicator of the melting transition.

\section{NOISE OF DYNAMICALLY "MELTED” SYSTEMS}

In this final section of the paper, we consider the similarities and differences between noise in the thermodynamic systems discussed above and noise in nonequilibrium systems. There are a very wide range of systems falling into the category of elastic media driven over periodic or random substrates, including friction where atoms move over a periodic potential created by the underlying stationary atoms,${ }^{34}$ sliding charge-density waves, ${ }^{35,36}$ and vortex motion in superconductors with random quenched disorder ${ }^{37,38}$ or periodic hole or dot arrays ${ }^{39-45}$

We add a term $\mathbf{f}_{d}=f_{d} \hat{x}$ to Eqn. (1) for the vortices in the $1 \mathrm{D}$ vortex chain system described in Section 2. This represents a uniform driving force applied to all of the vortices in the system which, in the absence of quenched disorder, would cause them to move in the positive $x$ direction, along the chain. In an experiment, this force would arise when a current is applied to the sample, creating a Lorentz force on the vortices that causes them to move in the direction perpendicular to the applied current. In the absence of temperature, $T=0$, as we slowly increase the driving force $f_{d}$ we find that for low values of drive, $f_{d}<f_{c}$, all of the vortices in the system remain trapped by the quenched disorder and do not move. At the critical force $f_{c}$, the first onset of vortex motion occurs. This motion can be detected experimentally in the form of a voltage drop, since the normal core 


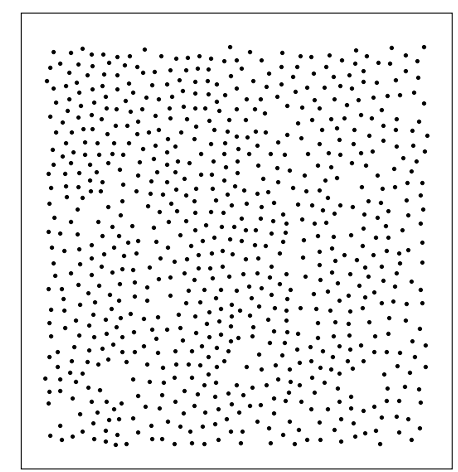

(a)

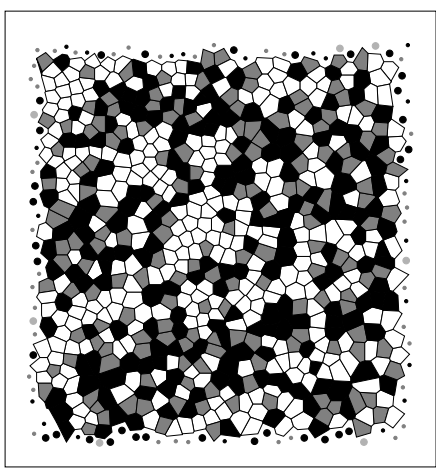

(b)

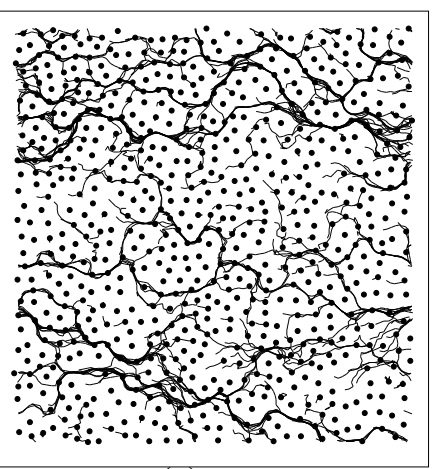

(c)

Figure 7. (a) Black dots: Colloids in the two-dimensional driven sample in the plastic flow regime. (b) Voronoi construction indicating sixfold (white), sevenfold (black), and fivefold (dark gray) coordinated particles. (c) Lines indicate colloid motion over a fixed time interval.

of each vortex dissipates energy when the vortex moves. The motion has also been observed directly in imaging experiments. ${ }^{46}$ In the case of the $1 \mathrm{D}$ vortex chain system, the entire system does not depin simultaneously. Instead, the vortices inside the 1D channel begin to move first, at a drive much lower than the drive at which the bulk vortices depin. This is similar to the melting transition shown in Section 2, where the vortices inside the channel begin to thermally diffuse at temperatures that are much lower than the temperature at which the bulk vortices begin diffusing. The motion of the $1 \mathrm{D}$ chain of vortices is the same as the motion shown in Fig. 1(c), with one important difference. In the thermally activated motion, the dislocations move diffusively to the left and right with equal likelihood, while in the driven motion, the dislocations move only to the right, giving a net transport of vortices in the direction of the drive. Just above the depinning force, $f_{d} \sim f_{c}$, the motion occurs as a soliton pulse, ${ }^{8-10,44,45}$ with each vortex jumping a single lattice constant and then remaining stationary most of the time, as illustrated in Fig. 3 of Ref. ${ }^{4}$ As in the case of the melting system, the disturbance or incommensuration moves much faster than the individual vortices. As the drive is further increased, however, more solitons appear and become more closely spaced, until at higher drives the motion of the vortices in the chain becomes continuous. At these high drives the velocity response of the vortices in the chain also becomes Ohmic and is linear with the drive.

Since there are similarities between the $1 \mathrm{D}$ system above melting and above depinning, it is interesting to ask whether similarities also occur for the $2 \mathrm{D}$ system. There has been a large amount of work exploring the dynamics of $2 \mathrm{D}$ driven vortex lattices interacting with disorder. Experiments, ${ }^{47-55}$ simulations, ${ }^{2,56-65}$ and theory ${ }^{37,38,56,66}$ suggest that at low drives the vortex lattice is disordered and exhibits plastic or random flow while at higher drives the lattice can undergo a reordering transition and flow elastically. In elastic flow, each particle maintains the same nearest neighbors, whereas in plastic flow, particles may move arbitrarily far away from their initial nearest neighbors and the lattice tears. In the highly driven state it was suggested by Koshelev and Vinokur ${ }^{56}$ that the flux lattice forms a moving crystal. This is due to the fact that, at high drives, the underlying quenched disorder begins to have an effect similar to temperature on the lattice, with the effective temperature decreasing as the lattice velocity increases. Thus, the lattice can freeze dynamically. Such dynamical reordering has been observed in simulations in vortex matter, ${ }^{2,63,65}$ charge-density wave systems, ${ }^{67}$ and driven Wigner crystals. ${ }^{3}$

We illustrate the motion of a $2 \mathrm{D}$ system of repulsive particles interacting with a random substrate at two different values of the driving force in Fig. 7 and Fig. 8. The system shown here is a colloidal sample with random pinning, interacting according to the potential given in Section 3 (see also Ref ${ }^{68}$ ); however, other elastic media including vortices and localized charges have been shown to produce the same behavior. ${ }^{2,3}$ At drives just above depinning, the flow is plastic as indicated in Fig. 7. Here the motion of the particles is clearly heterogeneous, with only riverlike channels of movement occurring at any given time. Similar channel structures, which resemble 


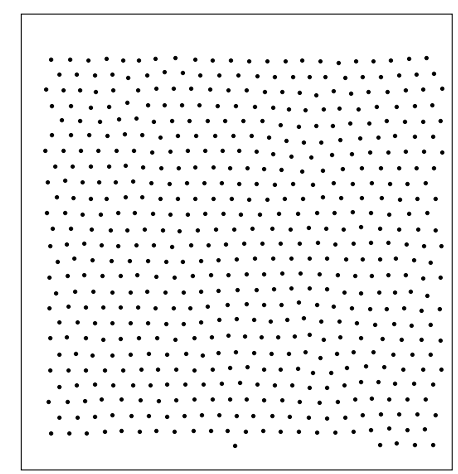

(a)

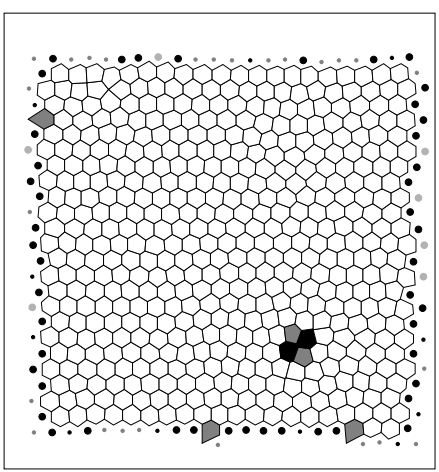

(b)

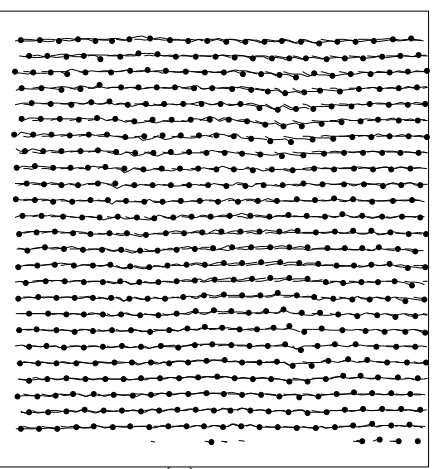

(c)

Figure 8. (a) Black dots: Colloids in the two-dimensional driven sample in the elastic flow regime. (b) Voronoi construction indicating sixfold (white), sevenfold (black), and fivefold (dark gray) coordinated particles. (c) Lines indicate colloid motion over a fixed time interval.

the fractal basins created by natural rivers ${ }^{69}$ have been observed in a large variety of systems, including fluid flow in a disordered landscape, Josephson junctions, Wigner crystals, magnetic bubbles, and stress networks in granular systems. The heterogeneity shows certain similarities to the heterogeneity just above melting in Section 3; however, here there is a clear alignment of the paths of particle motion with the drive, providing a net transport of particles. It is interesting to note that, in the plastic flow regime, individual particles do not move continuously. Instead, a particle will generally move only about a lattice constant before displacing a particle from a pin and becoming trapped in the now empty pin. The second particle takes up the motion and again moves about a lattice constant. Such pulse-like transport has been observed in a vortex system at drives very close to depinning, and is imaged in Ref. ${ }^{70}$ Again, it may be useful to think of the continuous motion of a disturbance or soliton, rather than the jerky motion of individual particles, as controlling the dynamics of the system. How to identify this soliton is not as clear as in the case of the thermal system.

At high drives, the lattice reorders and returns nearly to a perfect crystalline state, as illustrated in Fig. 8. The similarity between decreasing the drive from very high values and increasing the temperature in a clean system from low values was indicated by Koshelev and Vinokur, ${ }^{56}$ although their theory holds only in the limit of high drives when dislocations have not entered the lattice. It would be very interesting to explore further the possible connections between a lattice that has been disordered by temperature, and one that has been disordered due to plastic flow.

One notable similarity to the thermal case can be found in the noise produced by a plastically flowing system. Fig. 9 shows the fraction of sixfold coordinated particles $P_{6}$ and the noise power $S_{0}$ obtained from the time series of the particle velocity in the $x$ direction as a function of driving force $f_{d}$, both taken from a $2 \mathrm{D}$ simulation of vortices described in Ref. ${ }^{2}$ Here, the average vortex velocity is the analog of an experimentally measured voltage signal. The dynamic reordering transition at high drives to an elastically flowing lattice occurs at a drive $f_{d} \approx 1.6$. The noise power shows a peak in the plastic flow regime, when the heterogeneity of the vortex motion is at its maximum. This peak is also associated with the greatest transverse wandering of the vortices as they move, indicating that the channel structure is highly braided. ${ }^{1}$ The noise spectra is broad throughout the plastic flow regime, with a $1 / f^{\alpha}$ form. This is quite similar to the behavior of the noise power of the fluctuations in the dislocation number in the thermal system considered in Section 3. In both the plastic flow regime for the driven system, and the thermal system just above melting, the dynamics are highly heterogeneous. A deeper exploration of the similarities and differences in these heterogeneous states may lead to new insights into both systems. 


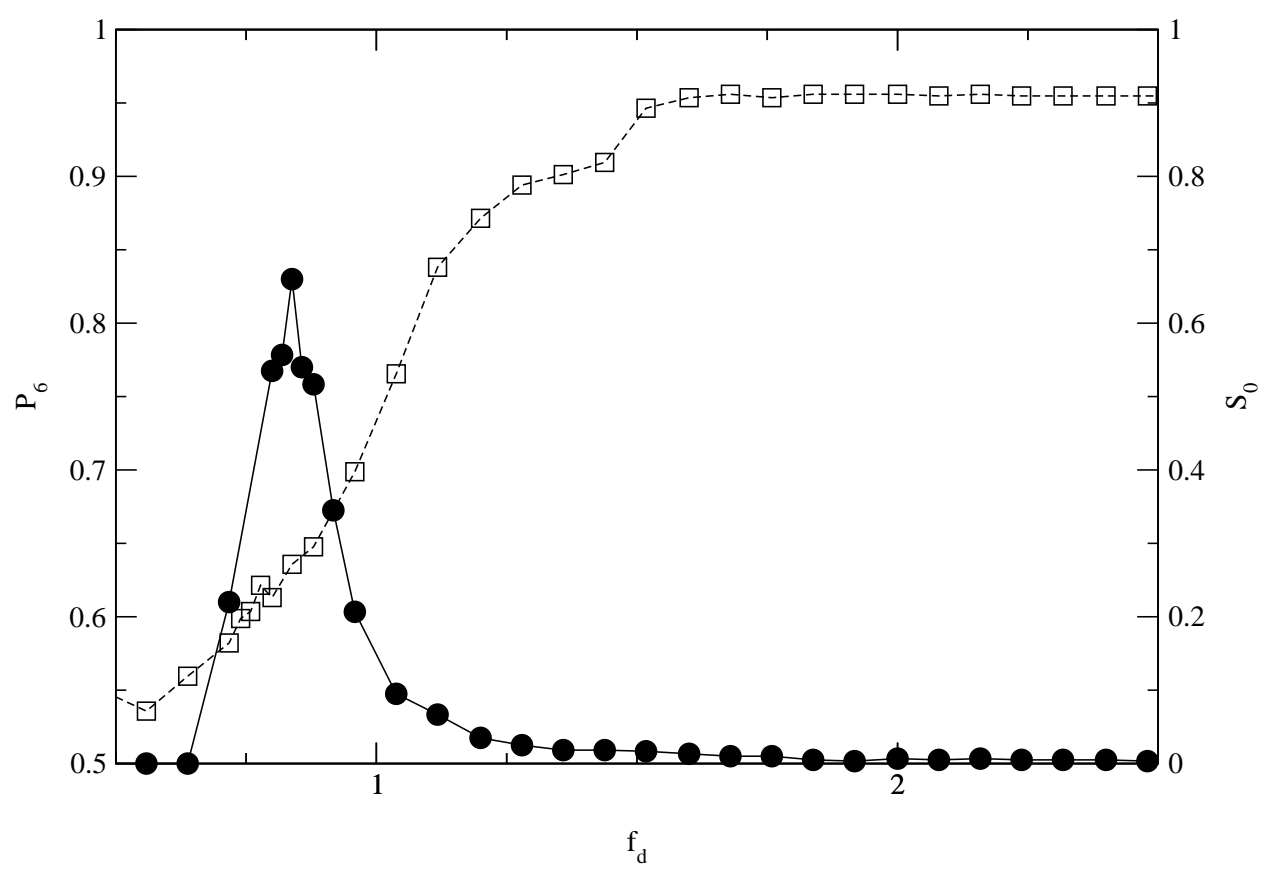

Figure 9. Squares (dotted line): The fraction of sixfold coordinated particles $P_{6}$ versus driving force $f_{d}$, with reordering occurring at $f_{d}=1.6$. Circles (solid line): The integrated noise power $S_{0}$, obtained from $S(f)$, shows a peak in the plastic flow region.

\section{CONCLUSION}

We have used noise measurements as a tool to explore the behavior of both thermally melted and dynamically disordered systems in 1D and 2D. We find that above a threshold, which is a melting transition in the thermal case, and a depinning transition in the driven case, the dynamics of the system becomes highly heterogeneous. In the thermal system, domains of ordered particles are surrounded by fluctuating grain boundaries, while in the driven system, regions of pinned particles are surrounded by riverlike filaments of moving particles. In each case, the motion of the particles is not continuous, but is characterized by long periods of no motion followed by a rapid change in position by about a lattice constant. In contrast, the motion of dislocations or solitons through the system is much more continuous, indicating that the particle motion can be considered as occurring whenever a dislocation goes past. The power spectrum of a time series of the particle position, particle velocity, or dislocation density has a $1 / f^{\alpha}$ characteristic in this regime, and the noise power peaks close to melting or depinning. At higher drives or lower temperatures, the system reorders and the noise power drops. These behaviors are not specific to a particular system, as we have shown here by considering both superconducting vortices as well as colloids in our simulations. Our results suggest that there may be interesting parallels between the heterogeneous nature of the molten phase in the thermal system, and the plastically flowing phase in the driven system.

\section{ACKNOWLEDGMENTS}

This work was supported by the US Department of Energy under Contract No. W-7405-ENG-36.

\section{REFERENCES}

1. C.J. Olson, C. Reichhardt, and F. Nori, "Fractal networks, braiding channels, and voltage noise in intermittently flowing rivers of quantized magnetic flux," Phys. Rev. Lett. 80, pp. 2197-2200, 1998. 
2. C.J. Olson, C. Reichhardt, and F. Nori, "Nonequilibrium dynamic phase diagram for vortex lattices," Phys. Rev. Lett. 81, pp. 3757-3760, 1998.

3. C. Reichhardt, C.J. Olson, N. Grønbech-Jensen, and F. Nori, "Moving Wigner glasses and smectics: Dynamics of disordered Wigner crystals," Phys. Rev. Lett. 86, pp. 4354-4357, 2001.

4. C. Reichhardt and C.J. Olson Reichhardt, "Dynamical behaviors of quasi-one-dimensional vortex states: Possible applications to the vortex chain state," Phys. Rev. B 66, pp. 172504/1-4, 2002.

5. C. Reichhardt and C.J. Olson Reichhardt, "Fluctuating topological defects in 2D liquids: Heterogeneous motion and noise," Phys. Rev. Lett. 90, pp. 095504/1-4, 2003.

6. C. Reichhardt, C.J. Olson, I. Martin, and A.R. Bishop, "Depinning and dynamics of systems with competing interactions in quenched disorder," Europhys. Lett. 61, pp. 221-227, 2003.

7. C. Bechinger, M. Brunner, and P. Leiderer, "Phase behavior of two-dimensional colloidal systems in the presence of periodic light fields," Phys. Rev. Lett. 86, pp. 930-933, 2001.

8. A. Pruymboom, P.H. Kes, E. van der Drift, and S. Radelaar, "Flux-line shear through narrow constraints in superconducting films," Phys. Rev. Lett. 60, pp. 1430-1434, 1988.

9. M.H. Theunissen, E. Van der Drift, and P.H. Kes, "Size effects in flow of flux-line solids and liquids," Phys. Rev. Lett. 77, pp. 159-162, 1996.

10. R. Besseling, R. Niggebrugge, and P.H. Kes, "Transport properties of vortices in easy flow channels: a Frenkel-Kontorova study," Phys. Rev. Lett. 82, pp. 3144-3147, 1999.

11. S.A. Anders, A.W. Smith, R. Besseling, P.H. Kes, and H.M. Jaeger, "Commensurability effects and fluctuations of vortex flow in mesoscopic channels," Physica C 332, pp. 35-39, 2000.

12. S. Anders, A.W. Smith, R. Besseling, P.H. Kes, and H.M. Jaeger, "Static and dynamic shear response in ultrathin layers of vortex matter," Phys. Rev. B 62, pp. 15195-15199, 2000.

13. M.J. Hogg, F. Kahlmann, E.J. Tarte, Z.H. Barber, and J.E. Evetts, "Vortex channeling and the voltagecurrent characteristics of $\mathrm{YBa}_{2} \mathrm{Cu}_{3} \mathrm{O}_{7}$ low-angle grain boundaries," Appl. Phys. Lett. 78, pp. 1433-1435, 2001.

14. A. Gurevich, M.S. Rzchowski, G. Daniels, S. Patnaik, B.M. Hinaus, F. Carillo, F. Tafuri, and D.C. Larbalestier, "Flux flow of Abrikosov-Josephson vortices along grain boundaries in high-temperature superconductors," Phys. Rev. Lett. 88, pp. 097001/1-4, 2002.

15. C.A. Duran, P.L. Gammel, R. Wolfe, V.J. Fratello, D.J. Bishop, J.P. Rice, and D.M. Ginsberg, "Real-time imaging of the magnetic flux distribution in superconducting $\mathrm{YBa}_{2} \mathrm{Cu}_{3} \mathrm{O}_{7-\delta}$," Nature (London) 357, pp. 474-477, 1992.

16. C.A. Bolle, P.L. Gammel, D.G. Grier, C.A. Murray, and D.J. Bishop, "Observation of a commensurate array of flux chains in tilted flux lattices in Bi-Sr-Ca-Cu-O single crystals," Phys. Rev. Lett. 66, pp. 112-115, 1991.

17. N. Grønbech-Jensen, "Summation of logarithmic interactions in periodic media," Int. J. Mod. Phys. C 7, pp. 873-881, 1996.

18. W. Kob, C. Donati, S.J. Plimpton, P.H. Poole, and S.C. Glotzer, "Dynamical heterogeneities in a supercooled Lennard-Jones liquid," Phys. Rev. Lett. 79, pp. 2827-2830, 1997.

19. C. Donati, J.F. Douglas, W. Kob, S.J. Plimpton, P.H. Poole, and S.C. Glotzer, "Stringlike cooperative motion in a supercooled liquid," Phys. Rev. Lett. 80, pp. 2338-2441, 1998.

20. M.M. Hurley and P. Harrowell, "Kinetic structure of a two-dimensional liquid," Phys. Rev. E 52, pp. 1694$1698,1995$.

21. W.K. Kegel and A. van Blaarderen, "Direct observation of dynamical heterogeneities in colloidal hard-sphere suspensions," Science 287, pp. 290-293, 2000.

22. E.R. Weeks, J.C. Crocker, A.C. Levitt, A. Schofield, and D.A. Weitz "Three-dimensional direct imaging of structural relaxation near the colloidal glass transition," Science 287, pp. 627-631, 2000.

23. B. Cui, B. Lin, and S.A. Rice, "Dynamical heterogeneity in a dense quasi-two-dimensional colloidal liquid," J. Chem. Phys. 114, pp. 9142-9155, 2001.

24. Y. Tang, A.J. Armstrong, R.C. Mockler, and W.J. O'Sullivan, "Free-expansion melting of a colloidal monolayer," Phys. Rev. Lett. 62, pp. 2401-2404, 1989. 
25. For reviews, see S.C. Glotzer, "Spatially heterogeneous dynamics in liquids: insights from simulation," $J$. Non-Cryst. Solids 274, pp. 342-355, 2000; R. Richert, "Heterogeneous dynamics in liquids: fluctuations in space and time," J. Phys. Condens. Matter 14, pp. R703-R738, 2002.

26. F.L. Somer, G.S. Canright, T. Kaplan, K. Chen, and M. Mostoller, "Inherent structures and two-stage melting in two dimensions," Phys. Rev. Lett. 79, pp. 3431-3434, 1997.

27. F.L. Somer, G.S. Canright, and T. Kaplan, "Defect-unbinding transitions and inherent structures in two dimensions," Phys. Rev. E 58, pp. 5748-5756, 1998.

28. A.H. Marcus, J. Schofield, and S.A. Rice, "Experimental observations of non-Gaussian behavior and stringlike cooperative dynamics in concentrated quasi-two-dimensional colloidal liquids," Phys. Rev. E 60, pp. 5725$5736,1999$.

29. R.A. Quinn and J. Goree, "Experimental test of two-dimensional melting through disclination unbinding," Phys. Rev. E 64, pp. 051404/1-10, 2001.

30. C.-H. Chiang and Lin I, "Cooperative particle motions and dynamical behaviors of free dislocations in strongly coupled quasi-2D dusty plasmas," Phys. Rev. Lett. 77, pp. 647-650, 1996.

31. W.T. Juan and Lin I, "Anomalous diffusion in strongly coupled quasi-2D dusty plasmas," Phys. Rev. Lett. 80, pp. 3073-3076, 1998.

32. R.D. Merithew, M.W. Rabin, M.B. Weissman, M.J. Higgins, and S. Bhattacharya, "Persistent metastable states in vortex flow at the peak effect in $\mathrm{NbSe}_{2}, "$ Phys. Rev. Lett. 77, pp. 3197-3199, 1996.

33. M.W. Rabin, R.D. Merithew, M.B. Weissman, M.J. Higgins, and S. Bhattacharya, "Noise probes of underlying static correlation lengths in the superconducting peak effect," Phys. Rev. B 57, pp. R720-R723, 1998.

34. B.N.J. Persson, Sliding Friction: Physical Principles and Applications, Springer, Heidelberg, 1998.

35. G. Grüner, "The dynamics of charge-density waves," Rev. Mod. Phys. 60, pp. 1129-1181, 1988.

36. R.E. Thorne, "Charge-density-wave conductors," Phys. Today 49(5), pp. 42-47, 1996.

37. L. Balents, M.C. Marchetti, and L. Radzihovsky, "Nonequilibrium steady states of driven periodic media," Phys. Rev. B 57, pp. 7705-7739, 1998.

38. P. LeDoussal and T. Giamarchi, "Moving glass theory of driven lattices with disorder," Phys. Rev. B 57, pp. 11356-11403, 1998.

39. M. Baert, V.V. Metlushko, R. Jonckheere, V.V. Moshchalkov, and Y. Bruynseraede, "Composite flux-line lattices stabilized in superconducting films by a regular array of artificial defects," Phys. Rev. Lett. 74, pp. 3269-3272, 1995.

40. K. Harada, O. Kamimura, H. Kasai, T. Matsuda, A. Tonomura, and V.V. Moshchalkov, "Direct observation of vortex dynamics in superconducting films with regular arrays of defects," Science 274, pp. 1167-1170, 1996.

41. J.I. Martin, M. Velez, J. Nogues, and I.K. Schuller, "Flux pinning in a superconductor by an array of submicrometer magnetic dots," Phys. Rev. Lett. 79, pp. 1929-1932, 1997.

42. L. Van Look, E. Rosseel, M.J. Van Bael, K. Temst, V.V. Moshchalkov, and Y. Bruynseraede, "Shapiro steps in a superconducting film with an antidot lattice," Phys. Rev. B 60, pp. R6998-R7000, 1999.

43. C. Reichhardt, R.T. Scalettar, G.T. Zimányi, and N. Grønbech-Jensen, "Phase-locking of vortex lattices interacting with periodic pinning," Phys. Rev. B 61, pp. R11914-R11917, 2000.

44. R. Surdeanu, R.J. Wijngaarden, R. Griessen, J. Einfeld, and R. Wördenweber, "Visualization of novel flux dynamics in $\mathrm{YBa}_{2} \mathrm{Cu}_{3} \mathrm{O}_{7-\delta}$ thin films with antidots," Europhys. Lett. 54, pp. 682-687, 2001.

45. C. Reichhardt, C.J. Olson, and F. Nori, "Nonequilibrium dynamic phases and plastic flow of driven vortex lattices in superconductors with periodic arrays of pinning sites," Phys. Rev. B 58, pp. 6534-6564, 1998.

46. T. Matsuda, O. Kamimura, H. Kasai, K. Harada, T. Yoshida, T. Akashi, A. Tonomura, Y. Nakayama, J. Shimoyama, K. Kishio, T. Hanaguri, and K. Kitazawa, "Oscillating rows of vortices in superconductors," Science 294, pp. 2136-2138, 2001.

47. S. Bhattacharya and M.J. Higgins, "Dynamics of a disordered flux line lattice," Phys. Rev. Lett. 70, pp. 2617-2620, 1993; A.C. Marley, M.J. Higgins, and S. Bhattacharya, "Flux flow noise and dynamical transitions in a flux line lattice," Phys. Rev. Lett. 74, pp. 3029-3032, 1995; M.J. Higgins and S. Bhattacharya, "Varieties of dynamics in a disordered flux-line lattice," Physica C 257, pp. 232-254, 1996. 
48. U. Yaron, P.L. Gammel, D.A. Huse, R.N. Kleiman, C.S. Oglesby, E. Bucher, B. Batlogg, D.J. Bishop, K. Mortensen, and K.N. Clausen, "Structural evidence for a two-step process in the depinning of the superconducting flux-line lattice," Nature (London) 376, pp. 753-755, 1995.

49. M.C. Hellerqvist, D. Ephron, W.R. White, M.R. Beasley, and A. Kapitulnik, "Vortex dynamics in twodimensional amorphous $\mathrm{Mo}_{7} 7 \mathrm{Ge}_{2} 3$ films," Phys. Rev. Lett. 76, pp. 4022-4025, 1996.

50. W. Henderson, E.Y. Andrei, M.J. Higgins, and S. Bhattacharya, "Metastability and glassy behavior of a driven flux-line lattice," Phys. Rev. Lett. 77, pp. 2077-2080, 1996.

51. A. Duarte, E.F. Righi, C.A. Bolle, F. de la Cruz, P.L. Gammel, C.S. Oglesby, E. Bucher, B. Batlogg, and D.J. Bishop, "Dynamically induced disorder in the vortex lattice of $2 \mathrm{H}-\mathrm{NbSe}_{2}$, " Phys. Rev. B 53, pp. 11336-11339, 1996.

52. F. Pardo, F. de la Cruz, P.L. Gammel, C.S. Oglesby, E. Bucher, B. Batlogg, and D.J. Bishop, "Topological defects in the flux-line lattice and their relationship to the critical current of a type-II superconductor," Phys. Rev. Lett. 78, pp. 4633-4636, 1997.

53. M. Marchevsky, J. Aarts, P.H. Kes, and M.V. Indenbom, "Observation of the correlated vortex flow in $\mathrm{NbSe}_{2}$ with magnetic decoration," Phys. Rev. Lett. 78, pp. 531-534, 1997.

54. F. Pardo, F. de la Cruz, P.L. Gammel, E. Bucher, and D.J. Bishop, "Observation of smectic and movingBragg-glass phases in flowing vortex lattices," Nature (London) 396, pp. 348-350, 1998.

55. A.M. Troyanovski, J. Aarts, and P.H. Kes, "Collective and plastic vortex motion in superconductors at high flux densities," Nature (London) 399, pp. 665-668, 1999.

56. A.E. Koshelev and V.M. Vinokur, "Dynamic melting of the vortex lattice," Phys. Rev. Lett. 73, pp. 3580$3583,1994$.

57. H.J. Jensen, A. Brass, and A.J. Berlinsky, "Lattice deformations and plastic flow through bottlenecks in a two-dimensional model for flux pinning in type-II superconductors," Phys. Rev. Lett. 60, pp. 1676-1679, 1988.

58. A.-C. Shi and A.J. Berlinsky, "Pinning and $I-V$ characteristics of a two-dimensional defective flux-line lattice," Phys. Rev. Lett. 67, pp. 1926-1929, 1991.

59. N. Grønbech-Jensen, A.R. Bishop, and D. Domínguez, "Metastable filamentary vortex flow in thin film superconductors," Phys. Rev. Lett. 76, pp. 2985-2988, 1996.

60. M.C. Faleski, M.C. Marchetti, and A.A. Middleton, "Vortex dynamics and defects in simulated flux flow," Phys. Rev. B 54, pp. 12427-12436, 1996.

61. S. Spencer and H.J. Jensen, "Absence of translational ordering in driven vortex lattices," Phys. Rev. B 55, pp. 8473-8481, 1997.

62. D. Domínguez, "Dynamic transition in vortex flow in strongly disordered Josephson junction arrays and superconducting thin films," Phys. Rev. Lett. 82, pp. 181-184, 1999.

63. K. Moon, R.T. Scalettar, and G.T. Zimányi, "Dynamical phases of driven vortex systems," Phys. Rev. Lett. 77, pp. 2778-2781, 1996.

64. S. Ryu, M. Hellerqvist, S. Doniach, A. Kapitulnik, and D. Stroud, "Dynamical phase transition in a driven disordered vortex lattice," Phys. Rev. Lett. 77, pp. 5114-5117, 1996.

65. A.B. Kolton, D. Domínguez, and N. Grønbech-Jensen, "Hall noise and transverse freezing in driven vortex lattices," Phys. Rev. Lett. 83, pp. 3061-3064, 1999.

66. S. Scheidl and V.M. Vinokur, "Driven dynamics of periodic elastic media in disorder," Phys. Rev. E 57, pp. 2574-2593, 1998.

67. L. Balents and M.P.A. Fisher, "Chiral surface states in the bulk quantum Hall effect," Phys. Rev. Lett. 76, pp. 2782-2785, 1996.

68. C. Reichhardt and C.J. Olson, "Colloidal dynamics on disordered substrates," Phys. Rev. Lett. 89, pp. 078301/1-4, 2002.

69. G. Korvin, Fractal Models in the Earth Sciences, Elsevier, Amsterdam, 1992.

70. C.J. Olson, C. Reichhardt, and F. Nori, "Superconducting vortex avalanches, voltage bursts, and vortex plastic flow: Effect of the microscopic pinning landscape on the macroscopic properties," Phys. Rev. B 56, pp. 6175-6195, 1997. 